\title{
Adaptive Antenna Null Broadening Beamforming against Array Calibration Error Based on Adaptive Variable Diagonal Loading
}

\author{
Wenxing Li, ${ }^{1}$ Yu Zhao, ${ }^{1}$ Qiubo Ye, ${ }^{2}$ and Bin Yang ${ }^{1}$ \\ ${ }^{1}$ College of Information and Communications Engineering, Harbin Engineering University, Harbin, China \\ ${ }^{2}$ Department of Electronics, Carleton University, Ottawa, ON, Canada \\ Correspondence should be addressed to Yu Zhao; zhaoyu0816@hrbeu.edu.cn
}

Received 7 June 2017; Accepted 29 August 2017; Published 8 October 2017

Academic Editor: Ikmo Park

Copyright (C) 2017 Wenxing Li et al. This is an open access article distributed under the Creative Commons Attribution License, which permits unrestricted use, distribution, and reproduction in any medium, provided the original work is properly cited.

\begin{abstract}
An approach for null broadening beamforming is proposed based on adaptive variable diagonal loading (VDL) and combined with the covariance matrix taper (CMT) approach, aiming at improving the robustness of adaptive antenna null broadening beamforming when array calibration error exists. Hence, it is named VDL-CMT. In this novel approach, the signal-to-noise ratio in the tapered sample covariance matrix is estimated and the VDL factor can be obtained adaptively. Then, the covariance matrix of the CMT approach is loaded with the obtained VDL factor. According to simulation results, in the case of array calibration error, robustness of the VDL-CMT is significantly improved and its performance is better than that of the existing adaptive antenna null broadening beamforming approaches.
\end{abstract}

\section{Introduction}

Adaptive antenna beamforming is a technique used to receive desired signals and suppress interferences by adjusting the weight vectors of arrays adaptively. It has been widely used in radar, wireless communication, radio astronomy, and many other areas [1-3]. The performance of the adaptive antenna beamforming will be severely degraded if the weight vector of an array cannot be able to adapt sufficiently fast to the jammer motion. Forming broad nulls around the directions of interferences is an effective means to settle this problem $[4,5]$.

There exist many adaptive antenna null broadening beamforming approaches in literature [6-14]. Covariance matrix taper (CMT) [6-9] is a classical and widespread approach of null broadening. The computational complexity of the CMT approach is extremely low, while the depth of broad nulls is shallow. Quadratic constraint sector suppressed (QCSS) $[10,11]$ is also a null broadening beamforming approach. The null depth of the QCSS is much deeper in comparison with CMT. However, the solving process of the QCSS approach is complicated. Linear constraint sector suppressed (LCSS) approach [12] is proposed on the basis of the QCSS approach. The nonlinear quadratic constraint is transformed into a set of linear constraints; hence the solving process is greatly simplified. A null broadening beamforming approach based on projection and diagonal loading (PDL) is proposed in [13], where the CMT and projection are combined. The null depth of the PDL approach is deeper than that of the CMT approach, and the computational complexity is low. The null broadening beamforming approach of covariance matrix reconstruction and similarity constraint (CMRSC) is proposed in [14]. The interference-plus-noise covariance matrix of array is reconstructed by the CMRSC. The depth of the broad nulls of CMRSC is much deeper than that of the CMT approach, but the computational complexity is high.

The approaches of LCSS [12], PDL [13], and CMRSC [14] are all proposed in recent years. Without array calibration error, deeper broad nulls and higher output signal-to-interference-plus-noise ratio (SINR) can be obtained by virtue of these approaches in comparison with the CMT approach. Nevertheless, all three approaches construct the correlation matrix of the steering vector based on the presumed steering vector, so they are greatly sensitive to array calibration error. In practice, the array calibration error may exist, such as the antenna location error and mutual coupling error [15]. In the case of the array calibration error, the performance of the 
above three adaptive antenna null broadening beamforming approaches will be severely degraded. On the contrary, the CMT approach [7] does not construct the correlation matrix of the steering vector. Therefore, its performance is better than that of the three approaches above in overcoming the array calibration error.

Diagonal loading (DL) [16] is a widespread robust beamforming technique. The robustness of adaptive antenna beamforming can be effectively improved by DL with a proper loading factor that is difficult to achieve $[17,18]$. The CMT approach always uses a small fixed loading factor $[9,13,14]$, so its robustness against the array calibration error is very limited. In order to improve the robustness of adaptive antenna null broadening beamforming against array calibration error, an adaptive variable diagonal loading based covariance matrix taper (VDL-CMT) null broadening beamforming approach is put forward. The signal-to-noise ratio (SNR) in the tapered sample covariance matrix is estimated and the variable diagonal loading (VDL) factor can be obtained adaptively. Then, the covariance matrix of the CMT approach is loaded with the obtained VDL factor, and the optimal weight vector of the adaptive antenna is calculated by the use of the loaded covariance matrix. The advancement and effectiveness of the VDL-CMT approach are verified by theoretical analysis and simulation results.

\section{The Existing Null Broadening Beamforming Approaches}

2.1. Signal Model. Consider a uniform linear array (ULA) with $N$ elements and the element spacing is equal to onehalf wavelength. $L$ is the number of far-field narrowband excitation sources which are incoherent. The received data $\mathbf{X}(t)$ of the antenna array can be expressed as

$$
\mathbf{X}(t)=\mathbf{A S}(t)+\mathbf{N}(t)
$$

where $\mathbf{A}=\left[\mathbf{a}_{1}(\theta), \mathbf{a}_{2}(\theta), \ldots, \mathbf{a}_{L}(\theta)\right]$ is the matrix of array steering vectors, $\mathbf{S}(t)$ is the complex sources envelope, and $\mathbf{N}(t)$ is the noise of the antenna array.

The covariance matrix of the array can be written as

$$
\mathbf{R}=\mathrm{E}\left\{\mathbf{X}(t) \mathbf{X}^{\mathrm{H}}(t)\right\}=\mathbf{A R}_{\mathrm{s}} \mathbf{A}^{\mathrm{H}}+\bar{\sigma}_{\mathrm{n}}^{2} \mathbf{I},
$$

where $\mathrm{E}\{\cdot\}$ denotes the expectation operation, $(\cdot)^{\mathrm{H}}$ denotes the conjugate transposition, $\mathbf{R}_{\mathrm{s}}$ represents the matrix of sources, $\bar{\sigma}_{\mathrm{n}}^{2}$ is the power of noise, and $\mathbf{I}$ is an identity matrix of $N$ dimension.

The standard minimum variance distortionless response (MVDR) beamformer can be expressed as follows:

$$
\begin{array}{cl}
\min _{\mathbf{w}} & \mathbf{W}^{\mathrm{H}} \mathbf{R}_{\mathbf{i}+\mathbf{n}} \mathbf{W} \\
\text { subject to } & \mathbf{W}^{\mathrm{H}} \mathbf{a}\left(\theta_{\mathrm{d}}\right)=1,
\end{array}
$$

where $\mathbf{W}$ is the weight vector of the array, $\mathbf{a}\left(\theta_{\mathrm{d}}\right)$ is the steering vector of the desired signal, and $\mathbf{R}_{\mathrm{i}+\mathrm{n}}$ is the covariance matrix of interference-plus-noise. Due to the difficulty of directly calculating $\mathbf{R}_{\mathrm{i}+\mathrm{n}}$, this matrix is commonly replaced by the sample covariance matrix with limited number of snapshots as

$$
\widehat{\mathbf{R}}=\frac{1}{K} \sum_{k=1}^{K} \mathbf{X}(k) \mathbf{X}^{\mathrm{H}}(k),
$$

where $K$ is the number of snapshots. The optimal weight vector of the MVDR beamformer can be expressed as

$$
\mathbf{W}_{\text {opt }}=\frac{\widehat{\mathbf{R}}^{-1} \mathbf{a}\left(\theta_{\mathrm{d}}\right)}{\mathbf{a}^{\mathrm{H}}\left(\theta_{\mathrm{d}}\right) \widehat{\mathbf{R}}^{-1} \mathbf{a}\left(\theta_{\mathrm{d}}\right)},
$$

where $(\cdot)^{-1}$ denotes the inverse operation. The output SINR is used to measure the output performance of the adaptive beamforming, which can be expressed as

$$
\operatorname{SINR}=\frac{\bar{\sigma}_{\mathrm{s}}^{2}\left|\mathbf{W}^{\mathrm{H}} \mathbf{a}\left(\theta_{\mathrm{d}}\right)\right|^{2}}{\mathbf{W}^{\mathrm{H}} \mathbf{R}_{\mathrm{i}+\mathrm{n}} \mathbf{W}}
$$

where $\bar{\sigma}_{\mathrm{s}}^{2}$ is the input power of the desired signal.

2.2. The CMT Approach. CMT [7] is a classical and widespread null broadening beamforming approach. The sample covariance matrix $\widehat{\mathbf{R}}$ is tapered by the matrix $\mathbf{T}_{\mathrm{CMT}}$ as

$$
\mathbf{R}_{\mathrm{CMT}}=\widehat{\mathbf{R}} \circ \mathbf{T}_{\mathrm{CMT}}
$$

where $\circ$ represents Hadamard product and $\mathbf{R}_{\mathrm{CMT}}$ denotes the tapered sample covariance matrix. The element of $m$ th row and $n$th column of $\mathbf{T}_{\mathrm{CMT}}$ can be expressed as

$$
T_{m n}=\frac{\sin ((m-n) \Delta)}{(m-n) \Delta}=\sin c\left(\frac{(m-n) \Delta}{\pi}\right),
$$

where $\sin c(x)=\sin (\pi x) / \pi x$, and $\Delta$ determines the width of the broad nulls. Then, the optimal weight vector of the array can be calculated using $\mathbf{R}_{\mathrm{CMT}}$.

The broad nulls can be formed around the interferences adaptively without the prior information about the directions of the interferences, and the computational complexity of the CMT approach is very low. However, a single interference is replaced by a set of virtual interferences with equal power to form the broad nulls, so the null depth of the CMT approach is shallow. This also leads to rising of sidelobes and deteriorates output performance.

2.3. The LCSS Approach. The average output power of the predefined sector $\Theta$ is constrained by the LCSS approach [12], which can be expressed as

$$
\mathbf{W}^{\mathrm{H}} \mathbf{Q W} \leq \eta,
$$

where $\eta$ is the predefined output power and $\mathbf{Q}$ is the correlation matrix of the steering vector that can be expressed as

$$
\mathbf{Q}=\int_{\Theta} \mathbf{a}(\theta) \mathbf{a}^{\mathrm{H}}(\theta) d \theta
$$


$S$ equidistant sampling points in sector $\Theta$ are used to construct $\mathbf{Q}$. The ideal goal to suppress the output power in sector $\Theta$ is to obtain a zero power response; that is, $\mathbf{W}^{\mathrm{H}} \mathbf{Q W}=0$. $\mathbf{Q}$ can be decomposed as $\mathbf{Q}=\mathbf{U} \boldsymbol{\Sigma} \mathbf{U}^{\mathrm{H}}$ based on its eigenvalues, where $\mathbf{U}$ is the matrix of eigenvectors and $\boldsymbol{\Sigma}$ is a diagonal matrix containing the eigenvalues of $\mathbf{Q}$ in a decreasing order. Assume that $\mathbf{Q}$ has a rank equal to $r$, and $\mathbf{U}_{r}=\left[\mathbf{u}_{1}, \ldots, \mathbf{u}_{r}\right]$ is the matrix of eigenvectors corresponding to the $r$ large eigenvalues. The quadratic constraint $\mathbf{W}^{\mathrm{H}} \mathbf{Q W}=0$ can be achieved if $\mathbf{W}^{\mathrm{H}} \mathbf{U}_{r}=\mathbf{0}$, so the broad nulls can be formed through a set of linear constraints as follows:

$$
\begin{array}{cl}
\min _{\mathbf{W}} & \mathbf{W}^{\mathrm{H}} \widehat{\mathbf{R}} \mathbf{W} \\
\text { subject to } & \mathbf{W}^{\mathrm{H}} \mathbf{C}=\mathbf{f}_{1}^{\mathrm{T}},
\end{array}
$$

where $\mathbf{C}=\left[\mathbf{a}\left(\theta_{\mathrm{d}}\right), \mathbf{U}_{r}\right], \mathbf{f}_{1}=\left[1, \mathbf{0}^{\mathrm{T}}\right]^{\mathrm{T}}$, and $(\cdot)^{\mathrm{T}}$ denotes transposition. The solution to (11) is

$$
\mathbf{W}_{\text {LCSS }}(r)=\widehat{\mathbf{R}}^{-1} \mathbf{C}\left(\mathbf{C}^{\mathrm{H}} \widehat{\mathbf{R}}^{-1} \mathbf{C}\right)^{-1} \mathbf{f}_{1},
$$

where $r$ is the number of linear constraints that minimize the average output power of sector $\Theta$. The depth of the broad nulls can be set according to the number of linear constraints. In the case without the array calibration error, the performance of the LCSS approach is better than that of the CMT approach. However, the correlation matrix of the steering vector in the LCSS approach is constructed based on the presumed steering vector and is very sensitive to the array calibration error. Therefore, the performance of the LCSS approach will be severely degraded when the array calibration error exists.

2.4. The PDL Approach. The broad nulls can be formed by the PDL approach [13] through projection. The correlation matrix of the steering vector is constructed in the predefined null broadening sectors as

$$
\mathbf{Z}_{\mathrm{P}}=\sum_{p=1}^{P} a\left(\theta_{p}\right) a^{\mathrm{H}}\left(\theta_{p}\right),
$$

where $P$ is the number of the predefined null broadening sectors. Combined with the CMT approach, $\mathbf{Z}_{\mathrm{P}}$ is tapered as

$$
\mathbf{R}_{\mathrm{P}}=\mathbf{Z}_{\mathrm{P}} \circ \mathbf{T}_{\mathrm{CMT}} .
$$

Based on its eigenvalues, $\mathbf{R}_{\mathrm{P}}$ is decomposed as

$$
\mathbf{R}_{\mathrm{P}}=\sum_{q=1}^{N} \lambda_{q} v_{q} v_{q}^{\mathrm{H}},
$$

where $\lambda_{q}$ is the eigenvalues of $\mathbf{R}_{\mathrm{P}}$, and $v_{q}$ is the corresponding eigenvectors. The projection matrix $\mathbf{T}_{\mathrm{P}}$ is constructed by the $M$ eigenvector corresponding to the large eigenvalues as

$$
\mathbf{T}_{\mathrm{P}}=\sum_{q=1}^{M} v_{q} v_{q}^{\mathrm{H}} .
$$

Then, we project $\mathbf{T}_{\mathrm{P}}$ onto the sample covariance matrix $\widehat{\mathbf{R}}$ as

$$
\mathbf{R}_{\mathrm{PDL}}=\mathbf{T}_{\mathrm{P}} \widehat{\mathbf{R}} \mathbf{T}_{\mathrm{P}}^{\mathrm{H}} .
$$

The optimal weight vector of the PDL approach can be calculated using $\mathbf{R}_{\mathrm{PDL}}$. The correlation matrix of the steering vector is constructed according to the presumed steering vector as formula (13) and very sensitive to the array calibration error. Thus the performance of the PDL approach will be severely degraded in the case of array calibration error.

2.5. The CMRSC Approach. The interference-plus-noise covariance matrix is reconstructed by the CMRSC approach [14] in the predefined sector $\Theta$, and the broad nulls can be formed as demonstrated below. Firstly, calculate the maximum power in sector $\Theta$ as the interference power, which can be expressed as

$$
\widetilde{\sigma}_{\mathrm{i}} \approx \max \left\{\frac{1}{\mathbf{a}^{\mathrm{H}}(\theta) \widehat{\mathbf{R}}^{-1} \mathbf{a}(\theta)}\right\}, \quad(\theta \in \Theta) .
$$

Reconstruct the interference covariance matrix according to $\widetilde{\sigma}_{\mathrm{i}}$ and the presumed steering vector as

$$
\widetilde{\mathbf{Q}}=\widetilde{\sigma}_{\mathrm{i}} \int_{\Theta} \mathbf{a}(\theta) \mathbf{a}^{\mathrm{H}}(\theta) d \theta .
$$

Then, $\widehat{\mathbf{R}}$ is decomposed based on its eigenvalues and the power of noise can be calculated according to the average value of the small eigenvalues corresponding to noise. The power of noise can be expressed as

$$
\widetilde{\sigma}_{\mathrm{n}}=\frac{1}{N-L} \sum_{q=L+1}^{N} \lambda_{q},
$$

where $L$ is the number of sources. The interference-plus-noise covariance matrix is reconstructed as

$$
\widetilde{\mathbf{R}}_{\mathrm{i}+\mathrm{n}}=\widetilde{\mathbf{Q}}+\widetilde{\sigma}_{\mathrm{n}} \mathbf{I} .
$$

The optimal weight vector can be calculated using $\widetilde{\mathbf{R}}_{\mathrm{i}+\mathrm{n}}$. The interference-plus-noise covariance matrix of the CMRSC approach is reconstructed according to the presumed steering vector as formula (19), so its performance will be severely degraded in the case of the calibration error.

\section{The Proposed Approach}

3.1. The Method to Obtain the VDL Factor. It is generally known that the improvement in robustness of the CMT approach by DL with a fixed loading factor is limited. The main reason is displayed in this paper by analyzing the effect of $\mathrm{DL}$ on the components of the tapered sample covariance matrix $\mathbf{R}_{\mathrm{CMT}}$, and then the VDL-CMT approach is proposed.

The covariance matrix of the CMT approach with DL can be expressed as $[7,9,16]$

$$
\begin{aligned}
\mathbf{R}_{\mathrm{CMT}} & =\widehat{\mathbf{R}} \circ \mathbf{T}_{\mathrm{CMT}}, \\
\mathbf{R}_{\mathrm{DL}} & =\mathbf{R}_{\mathrm{CMT}}+\alpha \sigma_{\mathrm{n}}^{2} \mathbf{I},
\end{aligned}
$$


where $\widehat{\mathbf{R}}$ is the sample covariance matrix, $\mathbf{I}$ is an identity matrix of $N$ dimension, and $\alpha$ is the loading factor that is expressed as the multiple of noise power $\sigma_{\mathrm{n}}^{2}$. $\mathbf{R}_{\mathrm{CMT}}$ is decomposed based on its eigenvalues as

$$
\mathbf{R}_{\mathrm{CMT}}=\mathbf{U} \boldsymbol{\Sigma} \mathbf{U}^{\mathrm{H}}=\mathbf{U}_{\mathrm{s}} \boldsymbol{\Sigma}_{\mathrm{s}} \mathbf{U}_{\mathrm{s}}^{\mathrm{H}}+\mathbf{U}_{\mathrm{n}} \boldsymbol{\Sigma}_{\mathrm{n}} \mathbf{U}_{\mathrm{n}}{ }^{\mathrm{H}},
$$

where $\mathbf{U}$ is the matrix of eigenvectors, $\boldsymbol{\Sigma}$ is a diagonal matrix containing the eigenvalues, $\mathbf{U}_{s}$ denotes the signal-plusinterference subspace, $\boldsymbol{\Sigma}_{\mathrm{s}}$ is a diagonal matrix containing the large eigenvalues corresponding to $\mathbf{U}_{s}, \mathbf{U}_{\mathrm{n}}$ denotes the noise subspace, and $\boldsymbol{\Sigma}_{\mathrm{n}}$ is a diagonal matrix containing the small eigenvalues corresponding to $\mathbf{U}_{\mathrm{n}} \cdot \mathbf{R}_{\mathrm{CMT}}$ can be expressed as

$$
\mathbf{R}_{\mathrm{CMT}}=\sigma_{\mathrm{d}}^{2} \mathbf{a}\left(\theta_{\mathrm{d}}\right) \mathbf{a}^{\mathrm{H}}\left(\theta_{\mathrm{d}}\right)+\sum_{i=1}^{L-1} \sigma_{i}^{2} \mathbf{a}\left(\theta_{i}\right) \mathbf{a}^{\mathrm{H}}\left(\theta_{i}\right)+\sigma_{\mathrm{n}}^{2} \mathbf{I},
$$

where $\sigma_{\mathrm{d}}^{2}$ is the power of the desired signal in $\mathbf{R}_{\mathrm{CMT}}, \sigma_{i}^{2}$ is the power of interference, $\sigma_{\mathrm{n}}^{2}$ is the power of noise, $\sigma_{\mathrm{d}}^{2} \mathbf{a}\left(\theta_{\mathrm{d}}\right) \mathbf{a}^{\mathrm{H}}\left(\theta_{\mathrm{d}}\right)$ denotes the desired signal component, $\sum_{i=1}^{L-1} \sigma_{i}^{2} \mathbf{a}\left(\theta_{i}\right) \mathbf{a}^{\mathrm{H}}\left(\theta_{i}\right)$ denotes the interference component, and $\sigma_{\mathrm{n}}^{2} \mathrm{I}$ denotes the noise component.

Ideally, $\mathbf{R}_{\mathrm{CMT}}$ can be obtained by tapering the interference-plus-noise covariance matrix $\mathbf{R}_{\mathrm{i}+\mathrm{n}}$, and the performance of the beamforming will be extremely good. However, $\mathbf{R}_{\mathrm{i}+\mathrm{n}}$ is commonly replaced by the sample covariance matrix $\widehat{\mathbf{R}}$ in practical applications [19]. The output performance and robustness of the CMT approach will be degraded, because $\widehat{\mathbf{R}}$ has two drawbacks compared with $\mathbf{R}_{\mathrm{i}+\mathrm{n}}$. Firstly, $\widehat{\mathbf{R}}$ is obtained through limited number of snapshots, which causes the disturbance of small eigenvalues corresponding to the noise subspace. The sidelobes will rise and the performance of the beamforming will be degraded [16, 17]. Secondly, $\widehat{\mathbf{R}}$ includes the desired signal component, which will degrade the performance of beamforming, especially in nonideal situations [19-21]. Therefore, if the disturbance of the small eigenvalues corresponding to the noise subspace is suppressed, the sidelobes will be depressed, and the output performance of beamforming will be improved. If the desired signal component or its proportion is decreased, the output performance and robustness of beamforming will be improved. The covariance matrix of the CMT approach with DL can be expressed as

$$
\begin{aligned}
\mathbf{R}_{\mathrm{DL}}= & \mathbf{R}_{\mathrm{CMT}}+\alpha \sigma_{\mathrm{n}}^{2} \mathbf{I} \\
= & \sigma_{\mathrm{d}}^{2} \mathbf{a}\left(\theta_{\mathrm{d}}\right) \mathbf{a}^{\mathrm{H}}\left(\theta_{\mathrm{d}}\right)+\sum_{i=1}^{L-1} \sigma_{i}^{2} \mathbf{a}\left(\theta_{i}\right) \mathbf{a}^{\mathrm{H}}\left(\theta_{i}\right)+\sigma_{\mathrm{n}}^{2} \mathbf{I} \\
& +\alpha \sigma_{\mathrm{n}}^{2} \mathbf{I} \\
= & \sigma_{\mathrm{d}}^{2} \mathbf{a}\left(\theta_{\mathrm{d}}\right) \mathbf{a}^{\mathrm{H}}\left(\theta_{\mathrm{d}}\right)+\sum_{i=1}^{L-1} \sigma_{i}^{2} \mathbf{a}\left(\theta_{i}\right) \mathbf{a}^{\mathrm{H}}\left(\theta_{i}\right) \\
& +\left(\sigma_{\mathrm{n}}^{2}+\alpha \sigma_{\mathrm{n}}^{2}\right) \mathbf{I} .
\end{aligned}
$$

It can be known from formula (25) that white noise is added to the covariance matrix by DL. The noise component is strengthened, exerting different impacts on each component of the covariance matrix. The disturbance of the small eigenvalues corresponding to the noise subspace is suppressed, so $\alpha$ should be bigger to further suppress the disturbance of the small eigenvalues. The SNR in the covariance matrix is decreased. That is to say, the proportion of the desired signal component is decreased. Therefore, $\alpha$ should be bigger to further reduce the SNR in the covariance matrix. The proportion of the interference component is decreased, which reduces the null depth, so $\alpha$ should be smaller to prevent the null depth from being reduced. Therefore, the components of $\mathbf{R}_{\mathrm{CMT}}$ have various requirements for $\alpha$, and they are contradictory. The performance improvement for the beamforming is limited if $\alpha$ is too small. On the contrary, the performance of the beamforming may be degraded if $\alpha$ is too large and so a proper loading factor is difficult to achieve. The CMT approach always uses a small fixed loading factor $[9,13,14]$. In the following, we make analysis on the main reason why the robustness improvement of the CMT approach by DL with a fixed loading factor is limited.

The SNR in $\mathbf{R}_{\mathrm{CMT}}$ is

$$
\begin{aligned}
& \beta=\frac{\sigma_{\mathrm{d}}^{2}}{\sigma_{\mathrm{n}}^{2}}, \\
& \gamma=10 \log _{10} \beta .
\end{aligned}
$$

The SNR in $\mathbf{R}_{\mathrm{DL}}$ is

$$
\begin{aligned}
& \beta_{\mathrm{DL}}=\frac{\sigma_{\mathrm{d}}^{2}}{\sigma_{\mathrm{n}}^{2}+\alpha \sigma_{\mathrm{n}}^{2}}=\frac{\beta}{1+\alpha}, \\
& \gamma_{\mathrm{DL}}=10 \log _{10} \beta_{\mathrm{DL}} .
\end{aligned}
$$

It can be obtained from formula (27) that if $\alpha$ is fixed, $\beta_{\mathrm{DL}}$ will always increase with the increase of $\beta$, so the SNR in the covariance matrix cannot be effectively decreased. That is the main reason why the robustness improvement of the CMT approach by DL with a fixed loading factor is limited. If $\alpha$ can be adjusted adaptively with the change of $\beta$, the performance of the CMT approach may be further improved. Thus the VDL-CMT approach is proposed in this paper, where the VDL factor $\alpha$ is adjusted adaptively according to $\beta$, and the SNR in the covariance matrix can be effectively decreased.

The minimum loading factor that can effectively decrease the SNR in the covariance matrix and suppress the disturbance of the small eigenvalues corresponding to the noise subspace is selected as the VDL factor for the VDL-CMT approach. When there is Gaussian white noise, the small eigenvalues disturb around the noise power $\sigma_{\mathrm{n}}^{2}$. The disturbance of the small eigenvalues can be partly suppressed when $\alpha \geq 2$. As the lower bound, 2 is a widely used loading factor, which can be used in both the MVDR beamforming and the CMT approach. In order to effectively decrease the SNR in the covariance matrix to a certain degree even if the input SNR is very high, $\alpha$ can be adjusted adaptively according to $\beta$ as

$$
\alpha \geq \frac{\sigma_{\mathrm{d}}^{2}}{\sigma_{\mathrm{n}}^{2}}=\beta .
$$


Formula (28) is obtained based on qualitative analysis. As the lower bound, when $\alpha$ is equal to $\beta$, the SNR in $\mathbf{R}_{\mathrm{DL}}$ can be expressed as

$$
\beta_{\mathrm{DL}}=\frac{\sigma_{\mathrm{d}}^{2}}{\sigma_{\mathrm{n}}^{2}+\sigma_{\mathrm{d}}^{2}}=\frac{\beta}{1+\beta} .
$$

It can be observed from formula (29) that, no matter how $\beta$ changes, there is always $\beta_{\mathrm{DL}}<1$ and $\gamma_{\mathrm{DL}}<0$. That is to say, the power of the desired signal in the covariance matrix is close to that of the noise. The SNR in the covariance matrix can be decreased effectively.

From the above analysis, we learn that the SNR in the covariance matrix can be effectively decreased when $\alpha \geq$ $\sigma_{\mathrm{d}}^{2} / \sigma_{\mathrm{n}}^{2}=\beta$, and the disturbance of the small eigenvalues corresponding to the noise subspace can be suppressed when $\alpha \geq 2$. The minimum VDL factor under the two constraints is a compromising $\alpha$ for the VDL-CMT approach, which can be expressed as

$$
\begin{aligned}
& \alpha=\frac{\sigma_{\mathrm{d}}^{2}}{\sigma_{\mathrm{n}}^{2}}=\beta, \quad(\beta>2), \\
& \alpha=2, \quad(\beta \leq 2) .
\end{aligned}
$$

The VDL factor $\alpha$ for the VDL-CMT approach is adjusted adaptively according to $\beta$, by which the SNR in the covariance matrix can be effectively decreased. Thus the robustness of the VDL-CMT approach is improved compared with the CMT approach using a fixed loading factor. It is important to emphasize that $\beta$ is the SNR in the tapered sample covariance matrix $\mathbf{R}_{\mathrm{CMT}}$, but not the input SNR of the array. Then, $\beta$ should be estimated to get $\alpha$.

3.2. The Method to Estimate $\beta$. There is no existing method to estimate $\beta$. In this paper, $\beta$ is obtained based on the relationship between $\beta$ and the SNR in the sample covariance matrix $\widehat{\mathbf{R}}$, which will be derived below.

Firstly, the SNR in $\widehat{\mathbf{R}}$ is estimated according to literature [22]. Similar to formula (23), $\widehat{\mathbf{R}}$ is decomposed based on its eigenvalues as

$$
\widehat{\mathbf{R}}=\sum_{q=1}^{N} \widehat{\lambda}_{q} \widehat{\boldsymbol{v}}_{q} \widehat{\boldsymbol{v}}_{q}^{\mathrm{H}}=\widehat{\mathbf{U}}_{\mathrm{s}} \widehat{\boldsymbol{\Sigma}}_{\mathrm{s}} \widehat{\mathbf{U}}_{\mathrm{s}}^{\mathrm{H}}+\widehat{\mathbf{U}}_{\mathrm{n}} \widehat{\Sigma}_{\mathrm{n}} \widehat{\mathbf{U}}_{\mathrm{n}}^{\mathrm{H}} .
$$

The presumed steering vector of the desired signal is $\mathbf{a}\left(\theta_{0}\right)$, which is projected to $\widehat{\boldsymbol{v}}_{q}$ as

$$
p(q)=\left|\widehat{\mathbf{v}}_{q}^{\mathrm{H}} \mathbf{a}\left(\theta_{0}\right)\right|^{2}
$$

$p(q)$ is sorted in a decreasing order as $p_{[N]} \geq \cdots \geq p_{[1]}$. The eigenvectors and the eigenvalues are all sorted corresponding to $p(q)$ as $\widehat{\mathbf{v}}_{[N]} \geq \cdots \geq \widehat{\mathbf{v}}_{[1]}$ and $\widehat{\lambda}_{[N]} \geq \cdots \geq \widehat{\lambda}_{[1]}$. The maximum of the projections $p_{[N]}$ is obtained when $\widehat{\boldsymbol{v}}_{q}$ is the eigenvector corresponding to the desired signal. The SNR in $\widehat{\mathbf{R}}$ can be expressed as [22]

$$
\begin{aligned}
& \widehat{\beta}=\frac{\widehat{\lambda}_{[N]}}{\widehat{\lambda}_{[1]}}, \\
& \widehat{\gamma}=10 \log _{10}\left(\frac{\widehat{\lambda}_{[N]}}{\widehat{\lambda}_{[1]}}\right) .
\end{aligned}
$$

Then, we derive the relationship between $\widehat{\beta}$ and $\beta$. According to formulas (18) and (19) in literature [9], $\mathbf{R}_{\mathrm{CMT}}$ can be expressed as

$$
\mathbf{R}_{\mathrm{CMT}}=\widehat{\mathbf{R}} \circ \mathbf{T}_{\mathrm{CMT}}=\widehat{\mathbf{R}} \circ \mathrm{E}\left\{\mathbf{e e}^{\mathrm{H}}\right\},
$$

where

$$
\mathbf{e}=\left[1, \ldots, e^{j n \omega}, \ldots, e^{j(N-1) \omega}\right]^{\mathrm{T}}
$$

where $-\Delta \leq \omega \leq \Delta$. When $\Delta=0$ and $\mathbf{e}=[1, \ldots, 1, \ldots, 1]^{\mathrm{T}}$, no broad nulls will be formed. When $\Delta>0$, the broad nulls will be formed with width $\Delta$, and $\mathbf{e}=\left[1, \ldots, e^{j n \omega}, \ldots, e^{j(N-1) \omega}\right]^{\mathrm{T}}$. Phase "dithered" is introduced into the steering vectors of $\widehat{\mathbf{R}}$ through e by the CMT approach [9]. According to formula (35), the original single phase becomes $N$ phases, which realizes the "dithered" phase. Therefore, the original single interference is replaced by $N$ virtual interferences with equal power, and broad nulls can be formed with the width $\Delta$. The desired signal is affected the same as interferences, so the relationship between $\beta$ and $\widehat{\beta}$ is

$$
\beta=\frac{\widehat{\beta}}{N}
$$

$\beta$ can be obtained according to formulas (33) and (36), so the VDL factor $\alpha$ for the adaptive VDL-CMT approach can be obtained according to $\beta$ and formula (30).

3.3. The VDL-CMT Approach. Solution procedures of the VDL-CMT approach are summarized as below.

Step 1. Obtain the tapered sample covariance matrix $\mathbf{R}_{\mathrm{CMT}}$ based on formulas (7) and (8).

Step 2. Estimate $\widehat{\beta}$ according to formulas (31), (32), and (33).

Step 3. Estimate $\beta$ according to formula (36).

Step 4. Obtain $\alpha$ according to formula (30).

Step 5. Obtain the loaded covariance matrix $\mathbf{R}_{\mathrm{DL}}$ according to $\alpha$ and formula (25).

Step 6. Calculate the optimal weight vector $\mathbf{W}_{\mathrm{opt}} \mathrm{using} \mathbf{R}_{\mathrm{DL}}$.

The VDL-CMT approach can be done by the above steps. The performance of the VDL-CMT approach is examined by simulations next. 


\section{Simulation Results}

Assume that the incoherent narrowband excitation sources are in the far field from the antenna array. We take account of a ULA with 12 array elements, and the element space is equal to one-half wavelength. The input SNR of array is $5 \mathrm{~dB}$. The input interference to noise ratio is $30 \mathrm{~dB}$. Additive noise is modeled as Gaussian white noise. The desired signal illuminates on the antenna array in the direction of $3^{\circ}$. The interferences are from the directions of $-40^{\circ}$ and $45^{\circ}$. Assume that the width of the broad nulls is set to $10^{\circ}$. The number of snapshots is 100. The VDL-CMT approach is compared with the LCSS approach [12], the PDL approach [13], the CMRSC approach [14], and the CMT approach [7] using the fixed loading factor 2. Four examples are used to verify the performance of the VDL-CMT approach. In all simulations, 100 Monte Carlo runs are used to obtain the results.

4.1. In the Case without Array Calibration Error. The presumed array steering vector is the same as the actual situation. Figure 1 shows the beam patterns obtained by the five approaches. Figure 2 shows the output SINR versus the input SNR for the five approaches and the theoretical optimal result when the input SNR changes.

It can be observed from Figure 1 that the broad nulls can be formed by the five approaches. The null depths of the LCSS, CMRSC, and PDL approaches are deeper than those of the VDL-CMT and CMT approaches. According to the presumed steering vector, the approaches of LCSS, CMRSC, and PDL construct the correlation matrixes of the steering vector and their performance is enhanced. It can be observed from Figure 2 that the output SINRs of the LCSS, CMRSC, and PDL approaches are all close to the theoretical optimal value. When the input $\mathrm{SNR}$ is lower than $0 \mathrm{~dB}$, the output SINRs of the VDL-CMT and CMT approaches are close to the theoretical optimal value. When the input SNR exceeds $0 \mathrm{~dB}$, the output SINR of the VDL-CMT approach is higher than that of the CMT approach. Consequently, without array calibration error, the performance of the LCSS, CMRSC, and PDL approaches is better than that of the VDL-CMT and CMT approaches. However in practice, array calibration error may exist, such as the antenna location error and mutual coupling error.

4.2. In the Case of Antenna Location Error. The difference between the presumed and actual location of each antenna element is modeled as a uniform random variable distributed in the interval $[-0.075 \lambda, 0.075 \lambda]$, where $\lambda$ is the wavelength. Figure 3 shows the beam patterns obtained by the five approaches. Figure 4 shows the output SINR versus the input SNR of the five approaches and the theoretical optimal result when the input SNR changes.

It can be observed from Figure 3 that the null positions of the LCSS, CMRSC, and PDL approaches have shifted. The three approaches construct the correlation matrix of the steering vector on the basis of the presumed steering vector. Therefore, they are sensitive to array calibration error. The broad nulls are formed by the approaches of VDL-CMT and

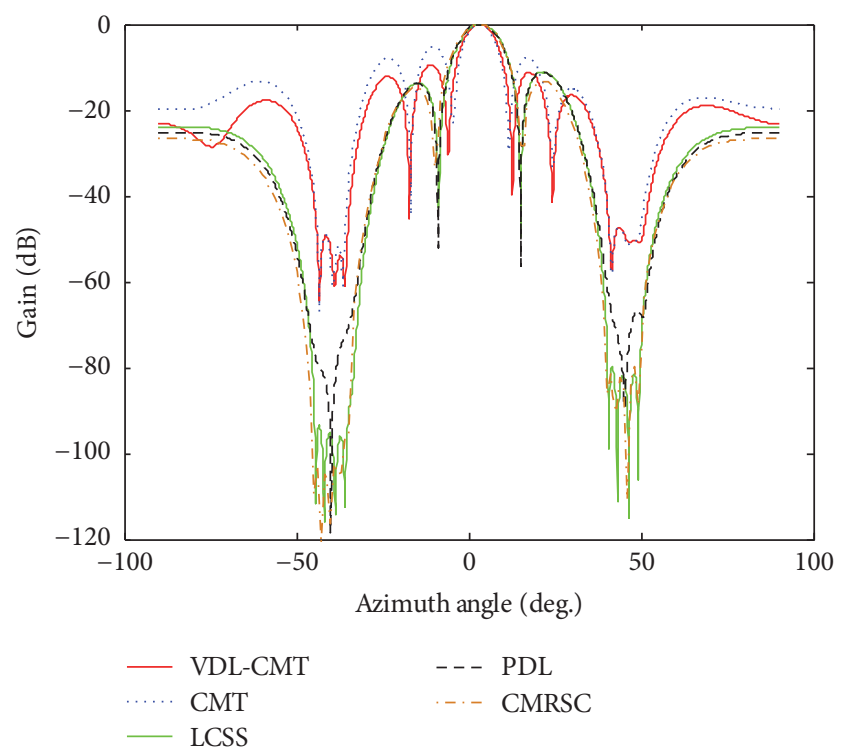

FIGURE 1: Beam patterns of the five approaches.

CMT and their robustness against the antenna location error is good. According to Figure 4, the output performance of the LCSS, CMRSC, and PDL approaches is severely degraded. The overall output performance of the VDL-CMT approach is better than that of other approaches. Therefore, the performance of the VDL-CMT approach is better than that of other adaptive null broadening beamforming approaches in the case of the antenna location error.

4.3. In the Case of Antenna Location Error and Mutual Coupling Error. Suppose that the mutual coupling only exists between adjacent array elements, and the coefficient of mutual coupling is $0.75 e^{-j \pi / 3}$. The remaining simulation conditions are the same as those in Section 4.2. Figure 5 shows the beam patterns obtained by the five approaches. Figure 6 shows the output SINR versus the input SNR of the five approaches and the theoretical optimal result when the input SNR changes.

It can be observed from Figure 5 that the null positions of the LCSS, CMRSC, and PDL approaches have shifted. The null positions of the CMT approach are accurate, but the main lobe is deformed. The VDL-CMT approach not only has accurate null positions but also has good performance on main lobe and sidelobes. The VDL-CMT approach adjusts the VDL factor adaptively to effectively decrease the SNR in the covariance matrix, so better robustness can be obtained compared with the CMT approach using a fixed loading factor. It can be observed from Figure 6 that the output performance of the approaches of LCSS, CMRSC, and PDL is severely degraded. The output SINR of the CMT approach decreases when the input SNR exceeds $0 \mathrm{~dB}$. The overall output performance of the VDL-CMT approach is better than that of other approaches, which shows that robustness against the array calibration error is improved in the VDLCMT approach. Therefore, the performance of the VDLCMT approach is better than that of other adaptive antenna 


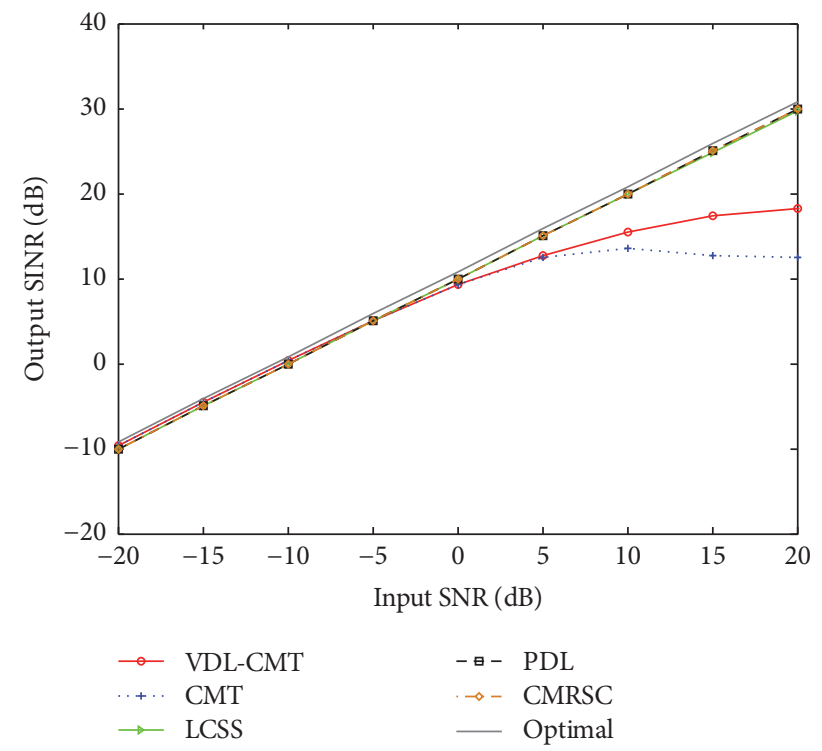

FIGURE 2: Output SINR versus input SNR of the five approaches and the theoretical optimal result.

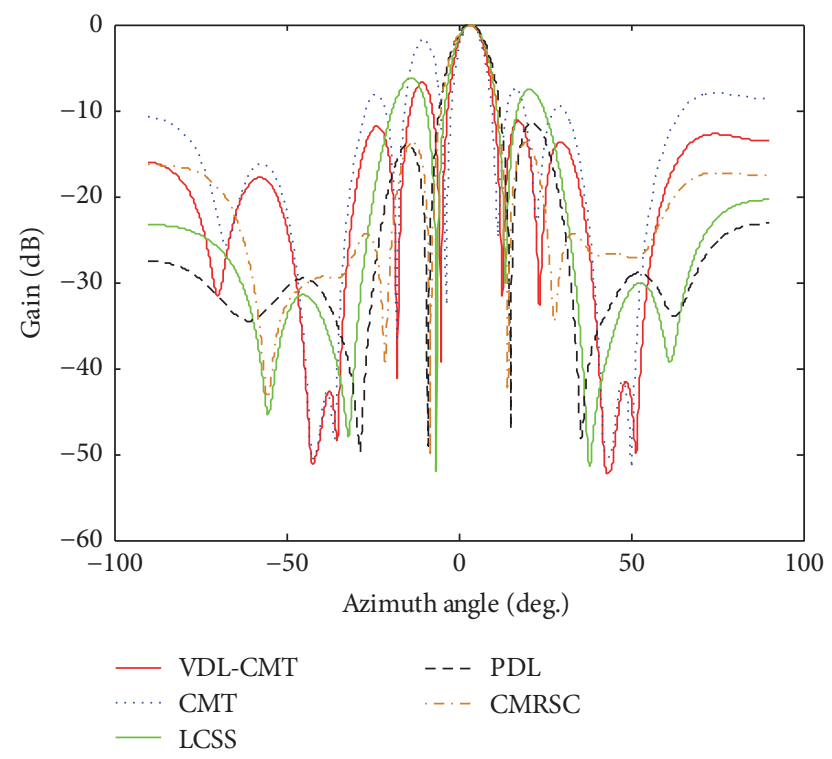

FIgURE 3: Beam patterns of the five approaches.

null broadening beamforming approaches in the case of the antenna location error and the mutual coupling error.

\subsection{In the Case of Antenna Location Error, Mutual Coupling} Error, and Jammer Motion. We assume that both of the interferences move. One interference moves from the $-40^{\circ}$ direction to the $0^{\circ}$ direction. The other interference moves from the $45^{\circ}$ direction to the $90^{\circ}$ direction. The moving speed is $0.03^{\circ}$ per snapshot. The remaining simulation conditions are the same as those in Section 4.3. Figure 7 shows the beam patterns obtained by the five approaches. Figure 8 shows the output SINR versus the input SNR of the five approaches and the theoretical optimal result when the input SNR changes.

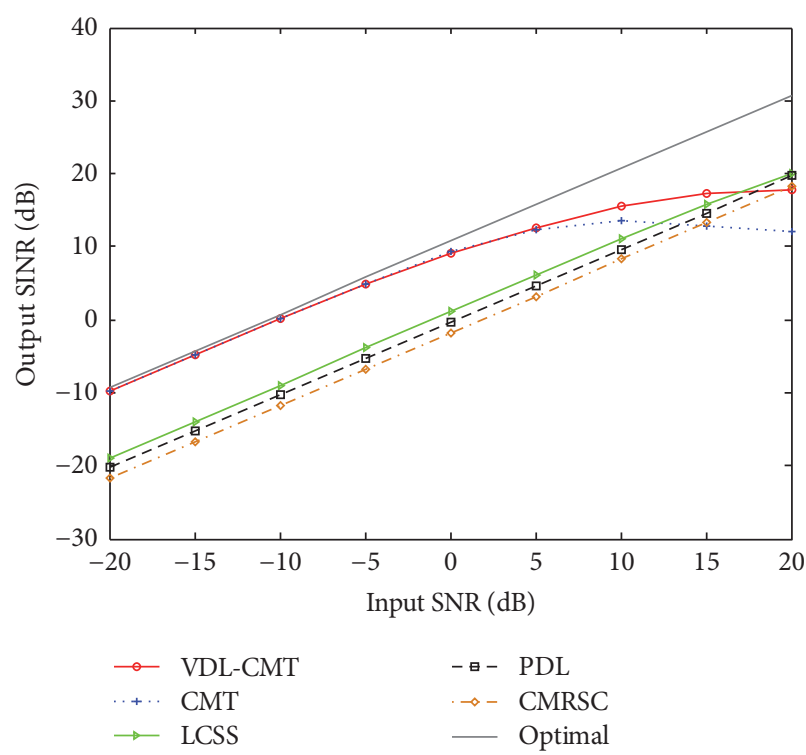

FIGURE 4: Output SINR versus input SNR of the five approaches and the theoretical optimal result.

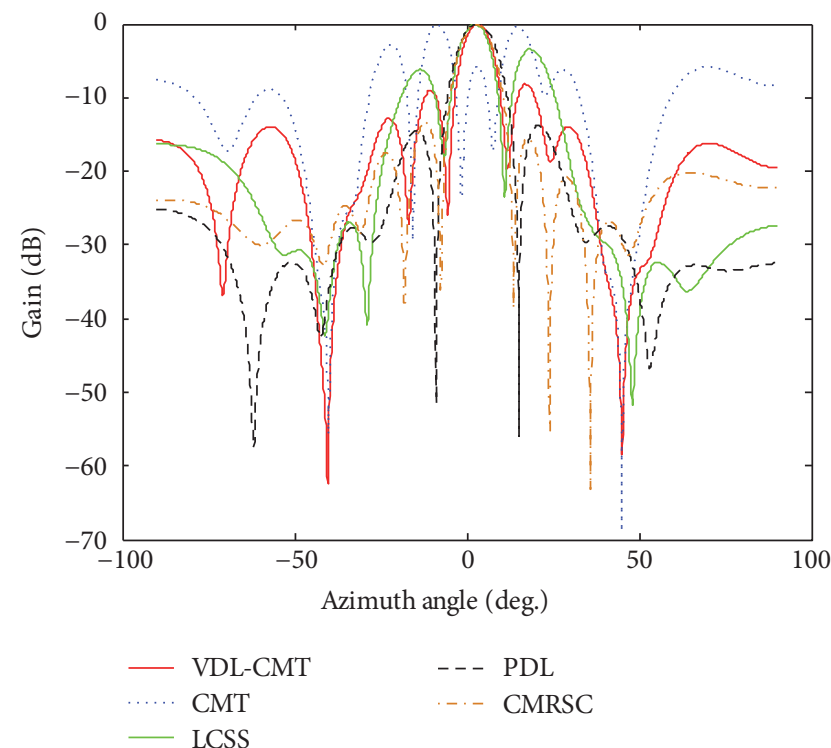

FIgURE 5: Beam patterns of the five approaches.

It can be observed from Figure 7 that the null positions of the LCSS, CMRSC, and PDL approaches have shifted. The null positions of the CMT approach are accurate, but the main lobe is deformed. The VDL-CMT approach not only has accurate null positions but also has good performance on main lobe and sidelobes. According to Figure 8, the output performance of the approaches of LCSS, CMRSC, and PDL is severely degraded. The output SINR of the CMT approach decreases when the input SNR exceeds $-5 \mathrm{~dB}$. The overall output performance of the VDL-CMT approach is better than that of other approaches. Therefore, when the antenna location error, the mutual coupling, and the jammer motion all exist, the performance of the VDL-CMT approach is better 


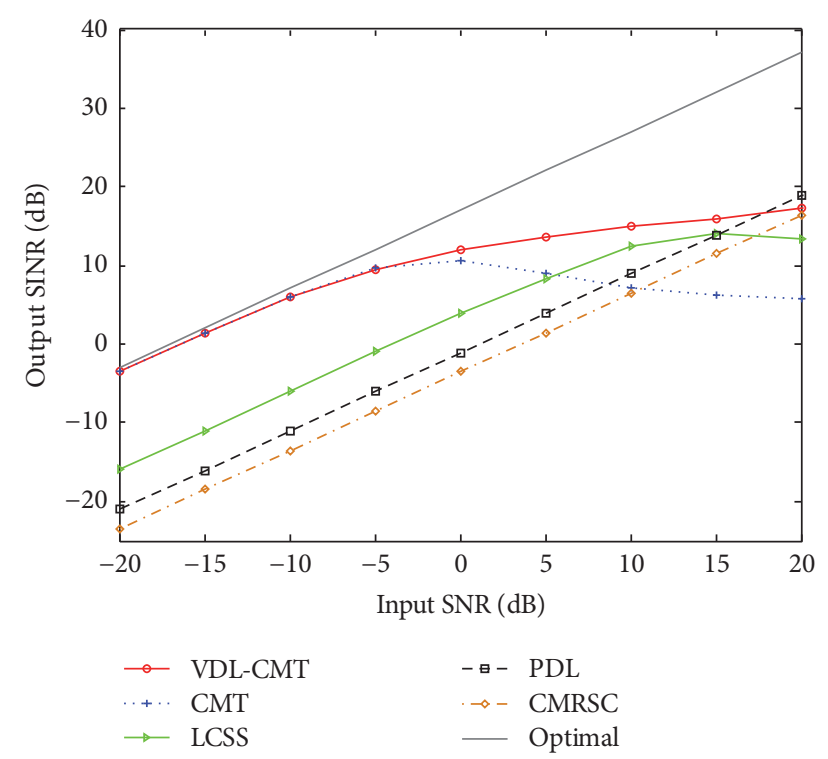

FIGURE 6: Output SINR versus input SNR of the five approaches and the theoretical optimal result.

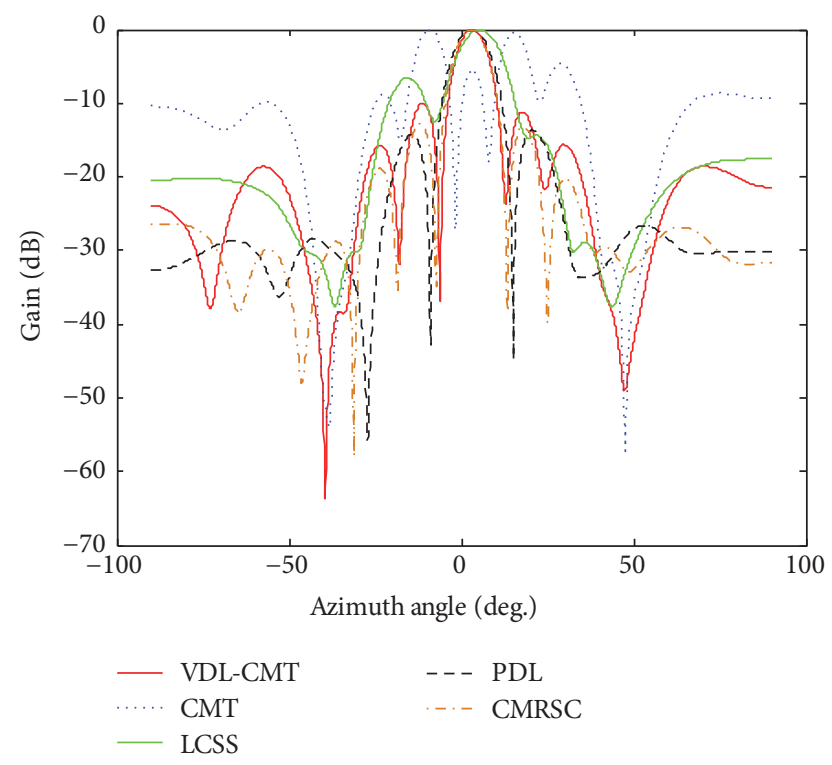

FIgURE 7: Beam patterns of the five approaches.

than that of the existing adaptive antenna null broadening approaches.

\section{Performance Comparison}

Firstly, the output performance is compared. Without the array calibration error, the performance of the LCSS, CMRSC, and PDL approaches is better than that of the VDLCMT and CMT approaches. The performance of the VDLCMT approach is better than that of other adaptive null broadening beamforming approaches in the case of the array calibration error.

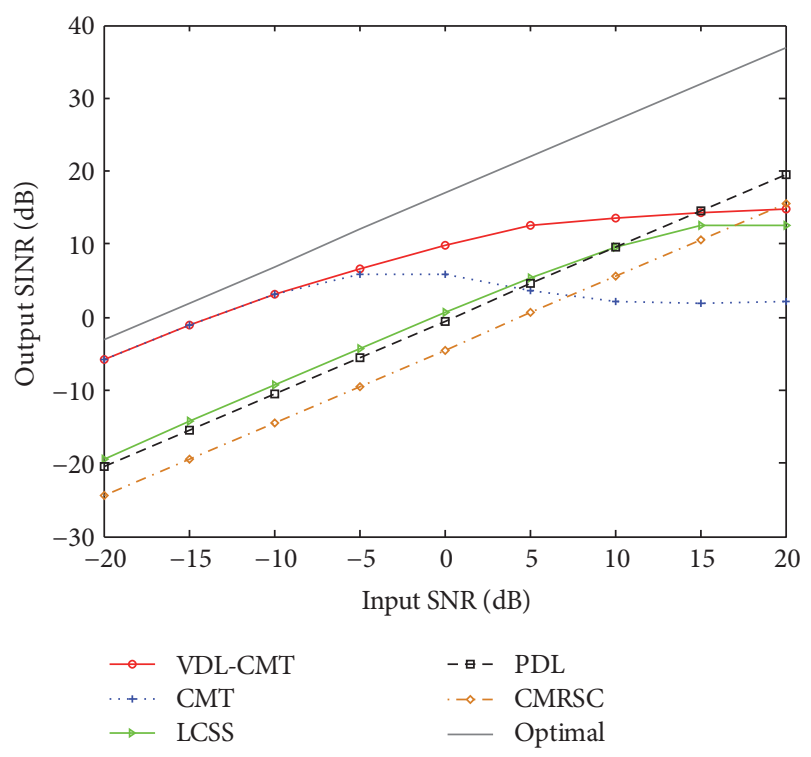

FIGURE 8: Output SINR versus input SNR of the five approaches and the theoretical optimal result.

Secondly, the computational complexity is compared. The computational complexity of the LCSS and CMRSC approaches is both $O\left(S N^{2}\right),(S \gg N)$, where $S$ is the number of sampling points in the predefined sector. The computational complexity of the PDL, VDL-CMT, and CMT approaches is all $\mathrm{O}\left(\mathrm{N}^{3}\right)$, and they are far below that of the LCSS and CMRSC approaches.

Lastly, the needed prior information is compared. The LCSS, CMRSC, and PDL approaches require the prior information about the directions of interferences. However, the VDL-CMT and CMT approaches do not need it.

\section{Conclusion}

The VDL-CMT approach is proposed through analyzing the reasons of the limited robustness of the CMT approach. It is derived that the VDL factor $\alpha$ can be adjusted adaptively according to $\beta$. It is shown by simulation results that the performance of the VDL-CMT approach is better than that of the existing adaptive antenna null broadening beamforming approaches in the case of the array calibration error. In addition, the jammer motion can be suppressed. Moreover, the computational complexity of the VDL-CMT approach is greatly low, and the VDL-CMT approach does not demand the prior information about the directions of interferences. Therefore, the VDL-CMT is an advanced and effective adaptive antenna null broadening beamforming approach that is capable of resisting to the array calibration error.

\section{Conflicts of Interest}

The authors declare that there are no conflicts of interest regarding the publication of this article. 


\section{Acknowledgments}

This paper was supported by the Pre-Research Fund of the 12th Five-Year Plan (no. 4010403020102). This paper is also supported by the Fundamental Research Funds for the Central Universities (nos. HEUCFD1433 and HEUCF1508).

\section{References}

[1] J. Yang, G. Liao, J. Li, Y. Lei, and X. Wang, "Robust beamforming with imprecise array geometry using steering vector estimation and interference covariance matrix reconstruction," Multidimensional Systems and Signal Processing, vol. 28, no. 2, pp. 451-469, 2017.

[2] W. Li, X. Mao, W. Yu, Y. Li, and C. Yue, "Robust adaptive array beamforming based on modified norm constraint algorithm," Applied Computational Electromagnetics Society Journal, vol. 29, no. 12, pp. 1060-1066, 2014.

[3] H. Li, K. Wang, C. Wang, Y. He, and X. Zhu, "Robust adaptive beamforming based on worst-case and norm constraint," International Journal of Antennas and Propagation, vol. 2015, Article ID 765385, 2015.

[4] M. S. Hossain, G. N. Milford, M. C. Reed, and L. C. Godara, "Robust efficient broadband antenna array pattern synthesis techniques," IEEE Transactions on Antennas and Propagation, vol. 62, no. 9, pp. 4537-4546, 2014.

[5] H. Song, W. A. Kuperman, W. S. Hodgkiss, P. Gerstoft, and J. S. Kim, "Null broadening with snapshot-deficient covariance matrices in passive sonar," IEEE Journal of Oceanic Engineering, vol. 28, no. 2, pp. 250-261, 2003.

[6] R. Mailloux, "Covariance matrix augmentation to produce adaptive array pattern troughs," Electronics Letters, vol. 31, no. 10, pp. 771-772, 1995.

[7] M. Zatman, "Production of adaptive array troughs by dispersion synthesis," Electronics Letters, vol. 31, no. 25, pp. 2141-2142, 1995.

[8] F. Liu, G. Sun, J. Wang, and R. Du, "Null broadening and sidelobe control algorithm via multi-parametric quadratic programming for robust adaptive beamforming," Applied Computational Electromagnetics Society Journal, vol. 29, no. 4, pp. 307315, 2014.

[9] J. R. Guerci, "Theory and application of covariance matrix tapers for robust adaptive beamforming," IEEE Transactions on Signal Processing, vol. 47, no. 4, pp. 977-985, 1999.

[10] M. H. Er, "Technique for antenna array pattern synthesis with controlled broad nulls," IEE Proceedings H: Microwaves, Antennas and Propagation, vol. 135, no. 6, pp. 375-380, 1988.

[11] L. C. Godara and M. R. S. Jahromi, "An optimized sector nulling technique for broadband antenna array," in Proceedings of the 2nd International Conference on Signal Processing and Communication Systems, ICSPCS 2008, aus, December 2008.

[12] A. Amar and M. A. Doron, "A linearly constrained minimum variance beamformer with a pre-specified suppression level over a pre-defined broad null sector," Signal Processing, vol. 109, pp. 165-171, 2015.

[13] X. Mao, W. Li, Y. Li, Y. Sun, and Z. Zhai, "Robust adaptive beamforming against signal steering vector mismatch and jammer motion," International Journal of Antennas and Propagation, vol. 2015, Article ID 780296, 12 pages, 2015.

[14] J. Qian, Z. He, J. Xie, and Y. Zhang, "Null broadening adaptive beamforming based on covariance matrix reconstruction and similarity constraint," Eurasip Journal on Advances in Signal Processing, vol. 2017, no. 1, article no. 1, 2017.
[15] L. Huang, J. Zhang, X. Xu, and Z. Ye, "Robust adaptive beamforming with a novel interference-plus-noise covariance matrix reconstruction method," IEEE Transactions on Signal Processing, vol. 63, no. 7, pp. 1643-1650, 2015.

[16] B. D. Carlson, "Covariance matrix estimation errors and diagonal loading in adaptive arrays," IEEE Transactions on Aerospace and Electronic Systems, vol. 24, no. 4, pp. 397-401, 1988.

[17] Y.-L. Chen and J.-H. Lee, "Finite data performance analysis of MVDR antenna array beamformers with diagonal loading," Progress in Electromagnetics Research, vol. 134, pp. 475-507, 2013.

[18] Y. Gu, N. A. Goodman, S. Hong, and Y. Li, "Robust adaptive beamforming based on interference covariance matrix sparse reconstruction," Signal Processing, vol. 96, pp. 375-381, 2014.

[19] S. A. Vorobyov, "Principles of minimum variance robust adaptive beamforming design," Signal Processing, vol. 93, no. 12, pp. 3264-3277, 2013.

[20] K. Yang, Z. Zhao, and Q. H. Liu, "Robust adaptive beamforming against array calibration errors," Progress in Electromagnetics Research, vol. 140, pp. 341-351, 2013.

[21] J. Li, G. Wei, and Y. Ding, "Adaptive beamforming based on covariance matrix reconstruction by exploiting interferences' cyclostationarity," Signal Processing, vol. 93, no. 9, pp. 25432547, 2013.

[22] W. Li, X. Mao, Z. Zhai, and Y. Li, "High performance robust adaptive beamforming in the presence of array imperfections," International Journal of Antennas and Propagation, vol. 2016, Article ID 3743509, 2016. 


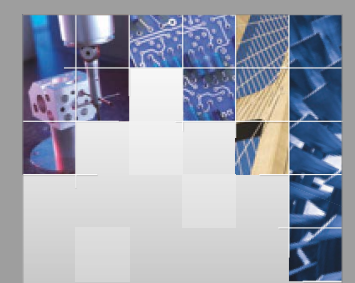

\section{Enfincering}
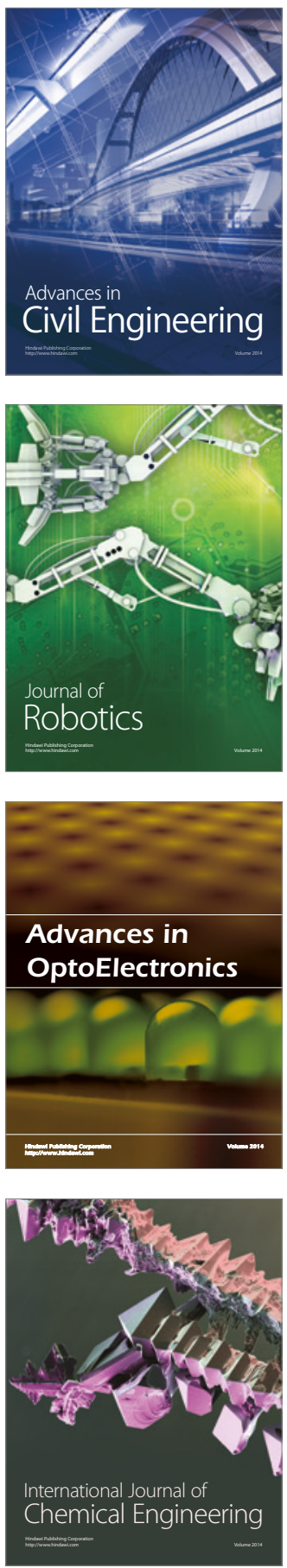

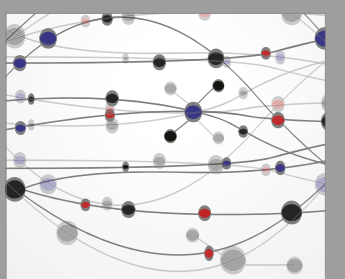

The Scientific World Journal

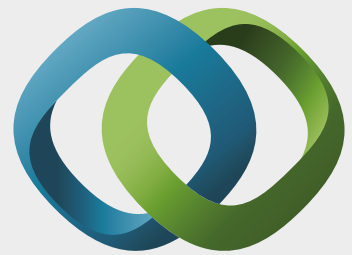

\section{Hindawi}

Submit your manuscripts at

https://www.hindawi.com
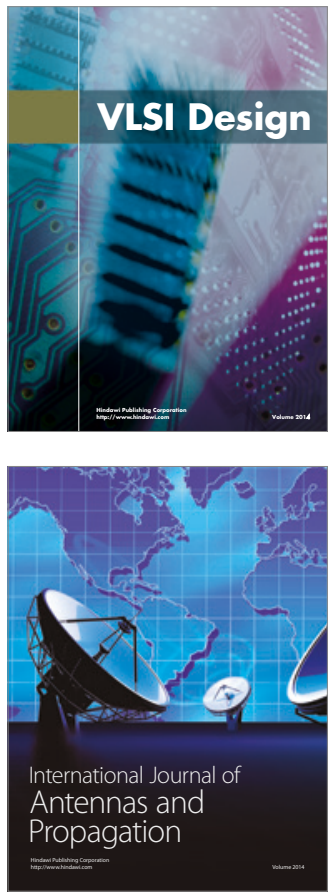

\section{Rotating}

Machinery
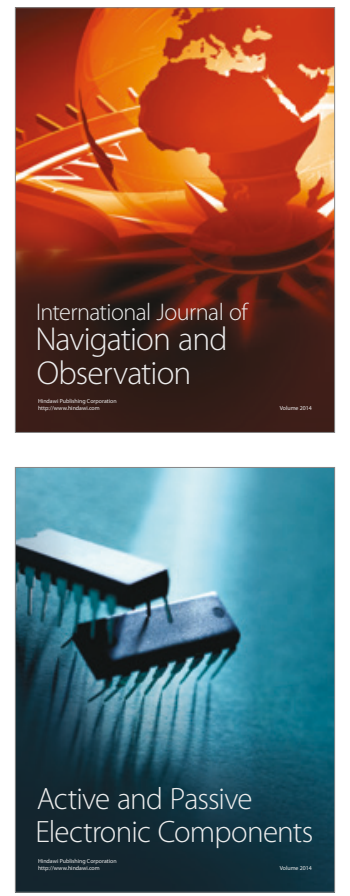
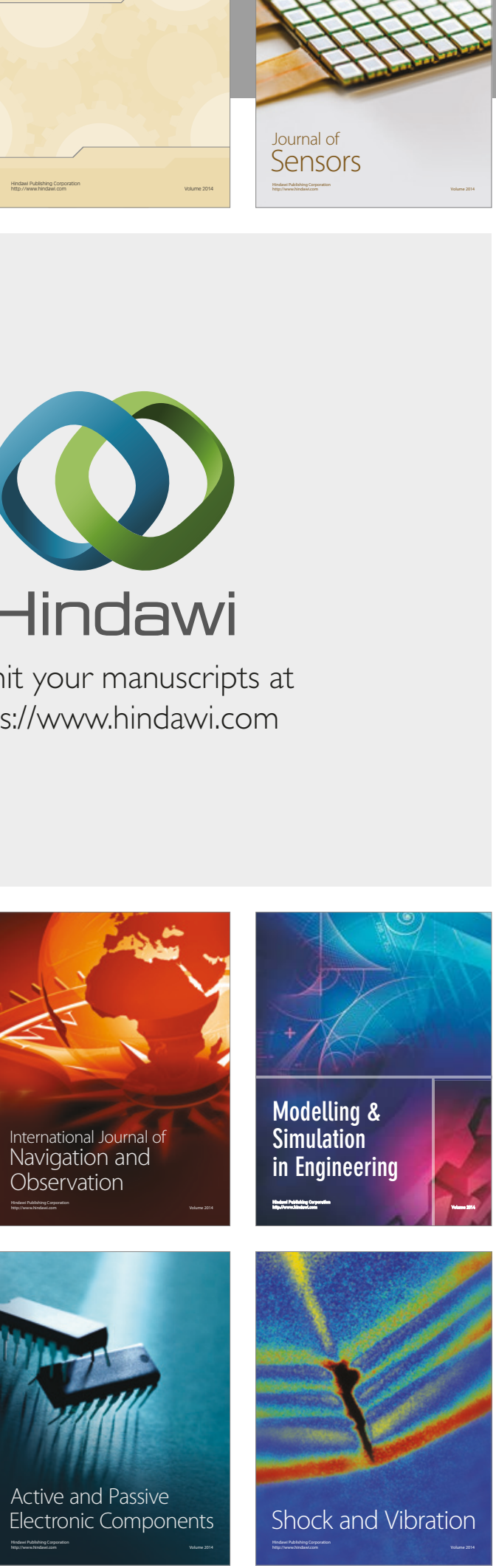
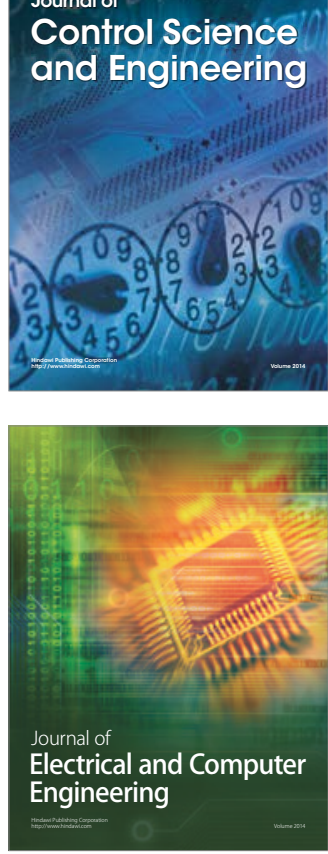

Distributed

Journal of

Control Science

and Engineering
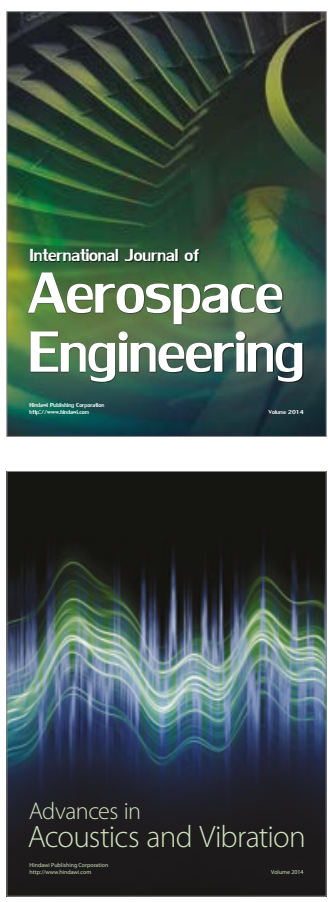

Sensor Networks 